\title{
Safety and Security specifications in an AR Context-Aware Systems: A model based on Bigraphical Reactive System(BRS)
}

\author{
Zahra Khoshmanesh \\ Department of Computer Science \\ Iowa State University \\ Ames, Iowa 50010 \\ Email: zkh@iastate.edu
}

\begin{abstract}
In context-awareness, the system behaves appropriately in different contexts. While context can be user-role, process-task, location, time and device, awareness based on location and user activity have been studied deeply in this field. Usage of context awareness in AR application is obvious since the system can sense or detects the contexts and help users by augmenting the reality of preciseness. In this study, we focus to model a context-aware specification for an AR application. We selected Bigraphical reactive system to formalize them since it has a very powerful natural system, which can help us effectively reason and validate the behavior of context-aware systems. Since nonfunctional properties such as security are rarely investigated, and they are most demanding, we limit our investigation to only specifications related to safety and security. We will formalize the context awareness of an AR smart car and investigate whether the bigraphical reactive system can help us to better visualize, analyze and trace the safety/security requirements. We conclude that the BRS can formalize much possible safety/security scenario in an augmented reality application. However, the AR application switches to different situations a lot and make the model be complicated and difficult to trace.
\end{abstract}

\section{Introduction}

Nowadays, We live in the world that computing found everywhere and anytime. The word ubiquitous computing is used to describe this pervasive computing. For example, in a smart car, multiple data from different sources such as GPS, camera, light sensors, traffic, and Wi-Fi are collected to compute and recognize hazardous situations in order to assist drivers. Mark Weiser, who introduced the ubiquitous computing term, suggested three preliminary form for the devices in ubiquitous system including "Tabs" or "wearable centimetre sized devices" like smartphone, "Pads" or "handheld decimetre-sized devices" like laptop, and "Boards" or "metre sized interactive display devices" like surface computers[1].

Later, context-awareness computing which originated from ubiquitous computing is introduced by Schilit[2] to describe the changes in a system behavior regarding the different contexts or situations. Dey[3] defines context as "any in- formation that can be used to characterize the situation of an entity." While in the first years of context-awareness 's emerging, context is considered the only location, contexts can be more than location. Dey and Abowd consider location, identity, activity and time as the context types[4]. Kaltz et al. categorize the contexts as userrole, processtask, location, time and device to cover a broad variety of mobile and web scenarios[5].

On the other hand, there is an increasing interest in using Augment Reality in different applications such as game, smart car and etc. Augmented Reality or AR is referred to the view of the physical world in such a way that the virtual concepts generated by computers combine with physical objects[6]. Augmented reality can be in the form of visual, auditory, haptic, somatosensory, and olfactory. In an augmented reality system, the system can take advantageous of ubiquitous computing taking place in different situations to enhance the perception of the physical environment for users. For instance, in the smart car augmented reality application, There are different situations that the smart car application must produce appropriate output regarding those situations. We can recognize different situations with using ubiquitous computing on different context's information such as being close to the pedestrian line or stop sign and consequently augment the driver's view to assisting him/her in avoiding hazardous situations.

While, there are different models to formalize the contextawareness system such as Context-Aware Calculus[7], Calculus of Context-Aware Ambient[8], and Bigraphical Reactive System[9], we selected Bigraphical Reactive System (BRS) to model the safety/security requirements in our augment reality system, a Smart Car since BRS integrates and takes benefit of both Calculus and graph notation.

The notion of bigraph introduced by Robin Milner in 2001. He invented the bigraph theory to model mobile interaction. In the bigraph theory, we have two graph structures: a graph referred topograph depicting locality and a graph referred edge net depicting connectivity[10]. The reaction of a system is expressed by a rule in the bigraph theory. We will explain the bigarph system with more details and an example in the background section.

The remainder of this paper is organized as follows: in Section 2, we present the background knowledge required 
for following the paper; in Section 3, we review the related works; in Section 4, we introduce our case study and express the study design; in section 5, we show the results and in Section 6, we discuss the future work and conclusion.

\section{Background}

In this section, we present the basic concepts of bigraph theory in reactive system with giving a base example of our study case that is the augmented reality system in the smart car. We used Big Red tool implemented by IT University of Copenhagen, Denmark available in Eclipse modeling framework. This tool supports the Bigraph Reactive System theory to draw and check the agents and reaction rules[11]. Each bigraph compose of two graphs: forest or place graph and hypergraph or link graph. Thus, we call it bigraph. In a bigraph with nodes $\mathrm{V}$ and edges $\mathrm{E}$, forest is a set of rooted tree and a hypergraph is a graph with nodes $\mathrm{V}$ and edges $\mathrm{E}$ [9]. Consider the bigraph reactive system of a smart car shown in Figure 1. The extracted place graph and link graph is depicted in Figure 2 and Figure 3 respectively.

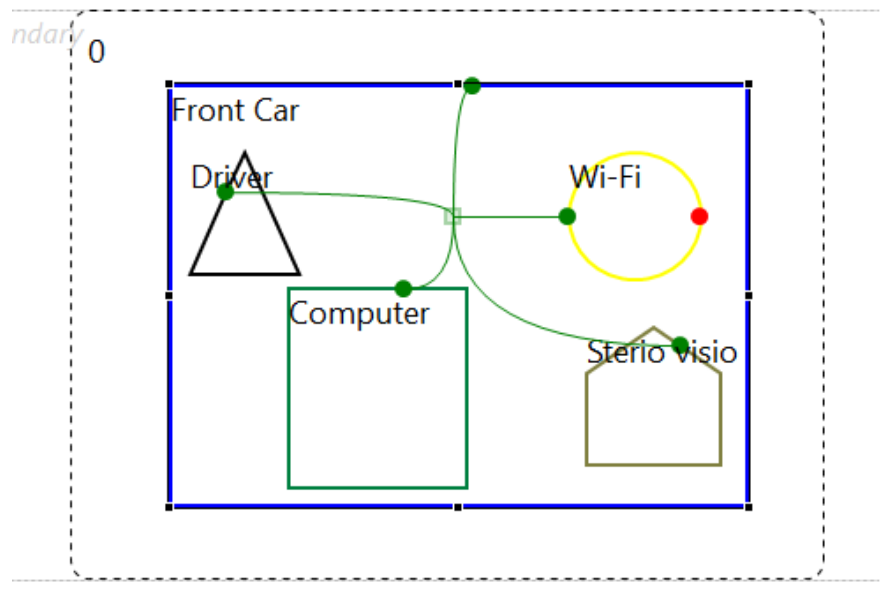

Figure 1. An example of a bigraph reactive system

In bigraph system, nodes are defined by their names or in general $v i$, edges are denoted by $e i$, the little dot that connect an edge and a node is called a port. For example in Figure 1 , Computer node has a port that connected the computer node with Driver which also has a port by the edge a. In the bigraph system, each node has a control that means the name or identity of an element.

And also we have a basic signature $(\mathrm{K}$, ar) in the bigraph system that assign a natural number, ar, to a control. for instance, the basic signature of the bigraph shown in figure 1 is $\mathrm{K}=\{$ FrontCar : 1, Driver : 2, Computer : $3, W i-F i: 4$, StereoVision : 5$\}$. In this example, the set of nodes are $\mathrm{V}=\{$ Front $\mathrm{Car}, \mathrm{Wi}-\mathrm{Fi}, \mathrm{Computer}$,Driver,Stereo Vision $\}$ and $\mathrm{E}=\left\{e_{0}\right\}$. Thus, in the place graph, we first visit the root 0 can be represent of a bigraph system. In the same

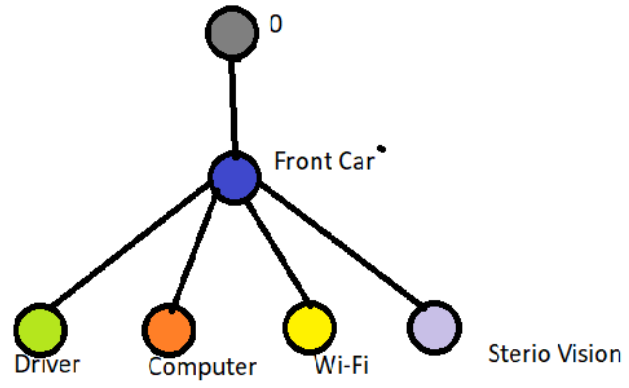

Figure 2. a place graph

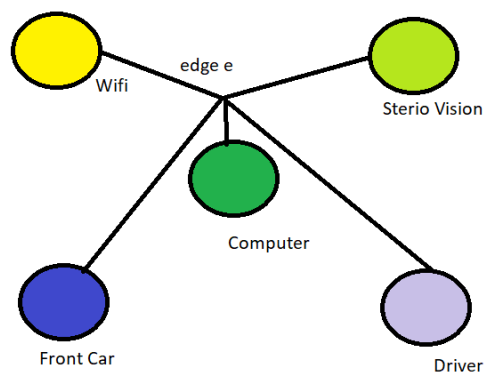

Figure 3. a link graph

way, you can see Front Car inside the root 0 and iteratively Driver, Computer, Stereo Vision, Wi-Fi inside the front car. Therefore they form a tree. In link graph, we connect the component based on the edge between them. Here, we have only one edge e that connect all elements to the computer.

For having a specific standard to draw and formalize the context-aware scenarios in a smart car augmented reality system, we are going to use the approach proposed in the study performed by Wang et al. to map between context elements and bigraphical component [16]. Table 1 shows the algebraic expression for bigraphs presented by Milner[9].

TABLE 1. THE ALGEBRIC EXPRESSION FOR BIGRAPHS

\begin{tabular}{|c|c|}
\hline Algebraic expression & Meaning \\
\hline$U \circ V$ & Composition of U and V \\
\hline$U \otimes V$ & Tensor product(juxtaposition of U and ) \\
\hline$U \| V$ & Parallel product(juxtaposition of roots) \\
\hline$U \mid V$ & Merge product(juxtaposition of children) \\
\hline$U \cdot V$ & Nesting(U contains V) \\
\hline$K_{x}$ & Ion. Node K whose ports linked to outer names $\mathrm{x}$ \\
\hline 1 & Site numbered i \\
\hline$d_{j}$ & Substitution \\
\hline$x / y$ & Plosure(replace outer name x of U with an edge) \\
\hline$/ x . U$ & Permution \\
\hline$\phi$ & \\
\hline$\pi$ & \\
\hline
\end{tabular}

In table 2, we extract and show the mapping relation between context-aware system elements and the bigraphical elements proposed by Wang et al.[16]. We can also show reaction rule by the bigraph system. Imagine we have two smart cars in the road.When rear car place in an accessible area of front car, they can connect with their Wi-Fi. In 
TABLE 2. THE ALGEBRIC EXPRESSION FOR BIGRAPHS

\begin{tabular}{|c|c|}
\hline Context-aware system element & Bigraph's component \\
\hline Physical Entity & Elliptic node \\
\hline Virtual Entity & Rectangular node \\
\hline Action & Hexagon node \\
\hline Information interaction & Edge \\
\hline Identity of entity & Control(name with number) \\
\hline Type of action & Control(name with number) \\
\hline Context-aware behavior & Reaction Rule \\
\hline
\end{tabular}

Figure 4 and 5, you can see this rule depicted by Big Red tool.

The algebric expression of the reaction rule shown in Figure 4 is as follows.

$\operatorname{Road}_{y} \cdot\left(\left(\right.\right.$ frontCar $\left._{m} . W i F i_{a}\right) \mid\left(\operatorname{RearCar}_{n} . W i F i_{b}\right)->$ /a.Roady. FrontCar $_{m}$. Wifi $i_{a} \mid$ RearCar $_{n}$. Wifi $\left._{a}\right)$

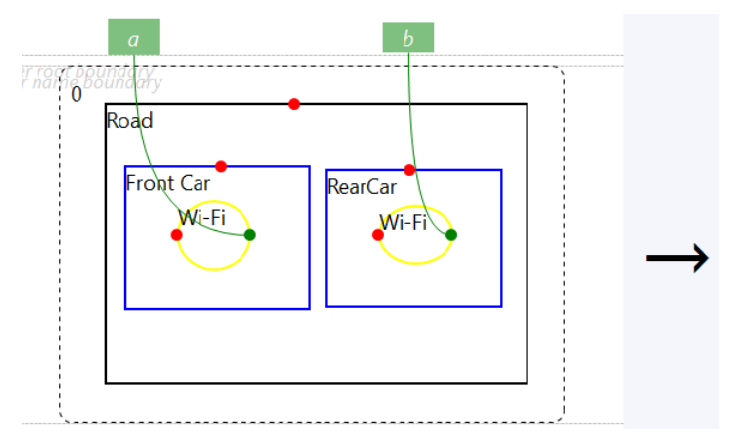

Figure 4. an example of an reaction rule(left side)

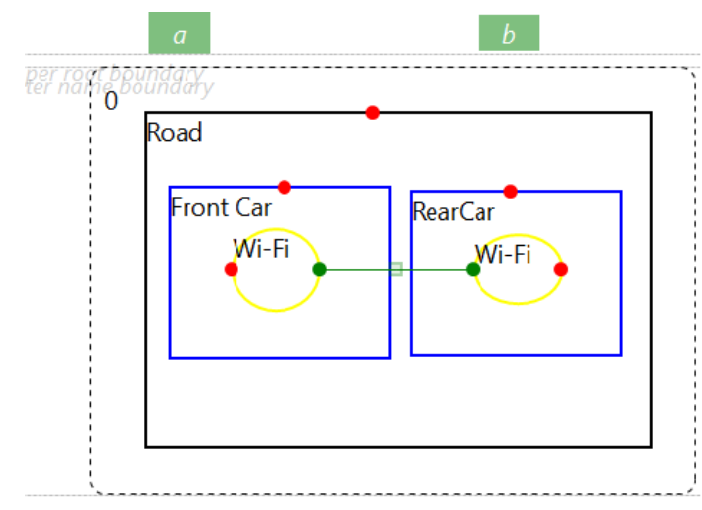

Figure 5. an example of an reaction rule(right side)

\section{Related Work}

Formalizing context-awareness are studied in a lot of works. They formalize it by using Calculus[7,12], languagebased model with variables[13], algebra[14] and bigraphical reactive system[9]. The bigraphical reactive system is presented to model the mobile agents communicate each other.The benefit of using BRS is to unify the calculus inside the graph model. One of the early study that used the BRS to model the context-awareness system proposed by Birkedal et al. in [15]. The authors in[16] formalize the structure and behavior of context-aware system in bigraph theory. They defined some sort of standard definition for elements of a bigraph system such as physical entity, virtual entity, Action, Information interaction, Identity of entity, Type of action, Context-aware behavior and propose an approach to map the mentioned elements of a context-aware scenario into bigraphical systems. However, they did not consider the safety/security scenarios and just provide a general view of a context-aware system.

The closest work to our study is performed in [17]. In this study, the authors formalize the context-awareness of a simple smart car by using the Bigraphical Reactive System. However, they just considered one scenario related to traffic sign to model the system. Also, they did not pay attention to possible safety/security scenarios in the system. Here, we want to concentrate and express the possible safety/security scenarios in a modern smart car formalizing with the Bigraphical Reactive System.

\section{Case Study}

We target three goals in our study as follows. First, we want to know what it can be possible safety/security scenarios in an augmented reality smart car. Second, we want to formalize them with using the Bigraphical Reactive System. Finally, we want to investigate whether the Bigraphical Reactive System is good choice to formalize the context-aware of an augmented reality application.

To address these issues we will center our study around answering the following research questions.

RQ1: What is the possible safety/security scenarios in the augmented reality smart car system?

RQ2:How effectively can we formalize the safety/security requirements in an augmented reality application with BRS?

\subsection{Software Subject Studied}

As we mentioned in the previous sections, we decided to formalize the context-awareness system of a smart car. We classified this system as an augmented reality system since all components inside a smart car provide appropriate data to the embedded computer in the car in order to create some virtual objects combining with physical objects that assist the driver to have safer and more secure driving.

\subsection{Study Design}

First, we discuss the possible safety/security scenarios in the smart car. We focus more to safety issues and identification security issues and formalize these scenario based on the bigraph reactive system explained in the background session. 


\subsection{The safety/security scenarios}

We will start counting the safety scenario with keeping in mind the 25 cause of car accidents reported in [18]. Among these causes, we believe that the augmented reality in the smart car can help driver to avoid large number of these causes. Some of these causes are distracted driving,speeding,reckless driving,rain,running red light, running stop signs, snow and Ice, night driving,wrong-way driving, improper turns, tailgating or too close to the car in front of, potholes, drowsy driving or being tired, tire blowouts, fog,curvy road, and construction zones. For example, in over speeding case, the augmented reality can show the virtual object in the screen of the smart car showing the limit speed. In this study, We will just go in deep in one of these scenarios since we want to make sure that the bigraph reactive system is enough good to visualize then expand our work..

Overtaking scenario: When a driver see a driver in front of him/her which drive slowly, the overtaking scenario happens. Since the driver has lack of visibility and cannot see in front of the car that wants to overtake, It cause to lead a hazardous situation that maybe cause an accident. Therefore, lack of visibility might be the reason for this hazardous situation.

The authors in[19] proposed a real-time augmented reality
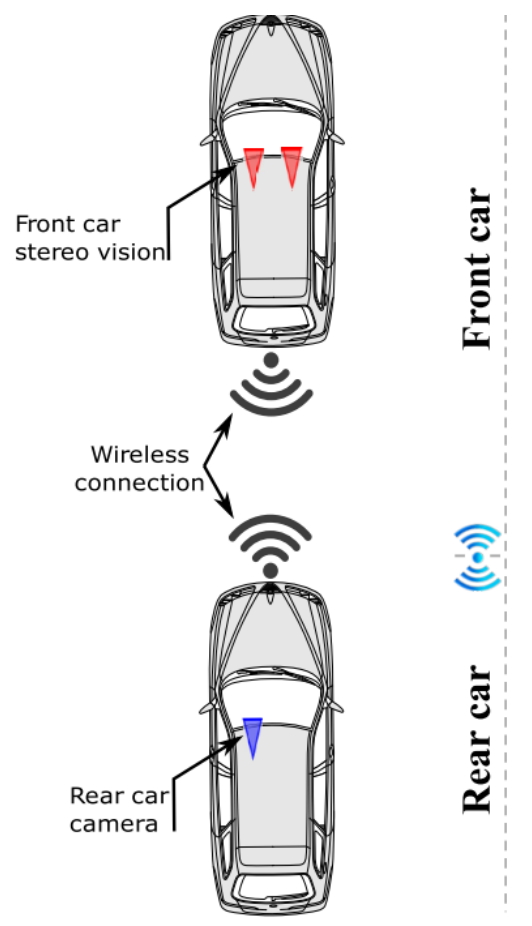

Figure 6. Overtaking scenario set up( extracted from[19]

system to See-Through cars to help the driver to avoid the hazardous scenario. We just consider the scenario and formalize it in the Bigraph reactive system. In Figure 6, the proposed scenario extracted from[19] is shown. In this scenario, the front car has a stereo vision that analyze and capture the view of the front car. The rear car connect via Wi-Fi, installed in both cars, to the front car. The front Car produce the appropriate field of view for rear car based on the its view. Finally, the rear view of the front car combined with some virtual objects is shown in the screen of the rear car. Thus, the rear car can see-through the front car and help him to safety overtake.

In figure 7 extracted again from[19], you can see the real field of view of rear car in the blue frame box and augmented field of view of rear car with help of the front car shown on the screen in the red frame box.

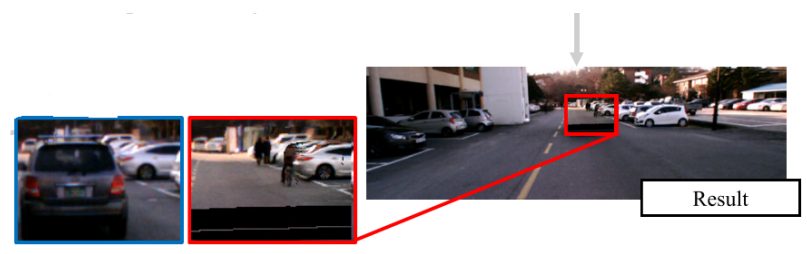

Figure 7. real vs augmented field of view( extracted from[19]

Formalize the overtaking scenario in BRS: To formalize the scenario in the Bigraphical Reactive System, we need toformalize the agents,signatures and reaction ruls. We use Big Red tool introduced in [11]. Figure 8 and Figure 9 shows the reaction rule based on the overtake scenario. In figure

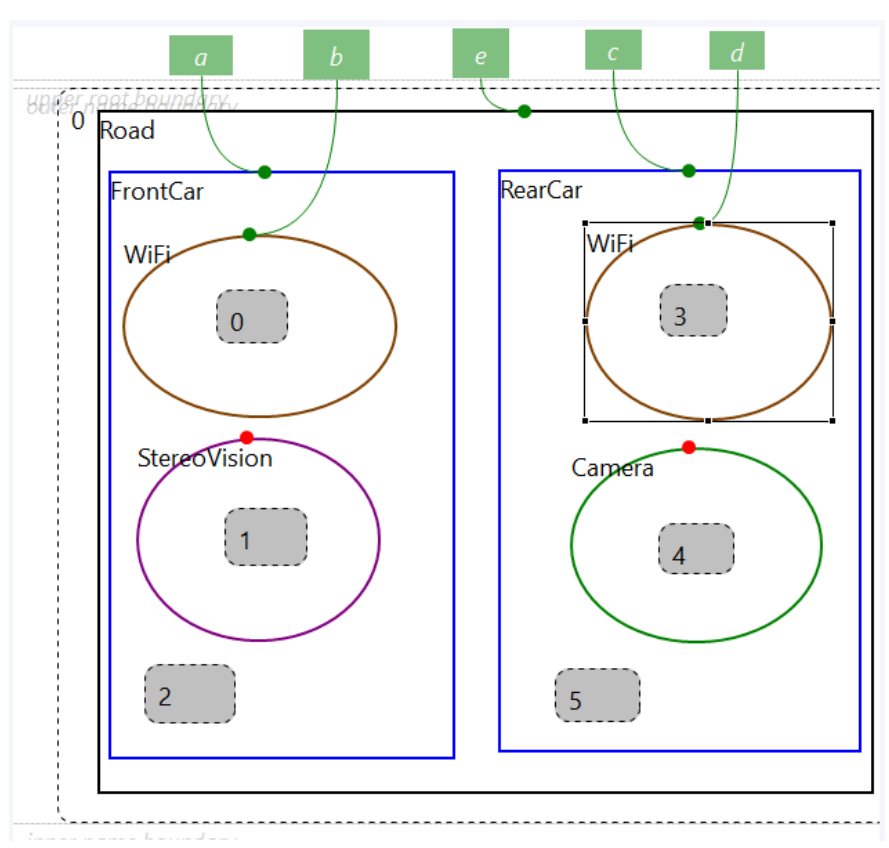

Figure 8 . overtaking scenario-left side of the rule

8, the situation of Front car and Rear car in the road are depicted. We have wifi and stereo vision system as physical objects inside the front car. We have also the site number assigned for every physical object representing the process happening in the site of the physical objects. Every physical objects can have an outer name means that they can interact 


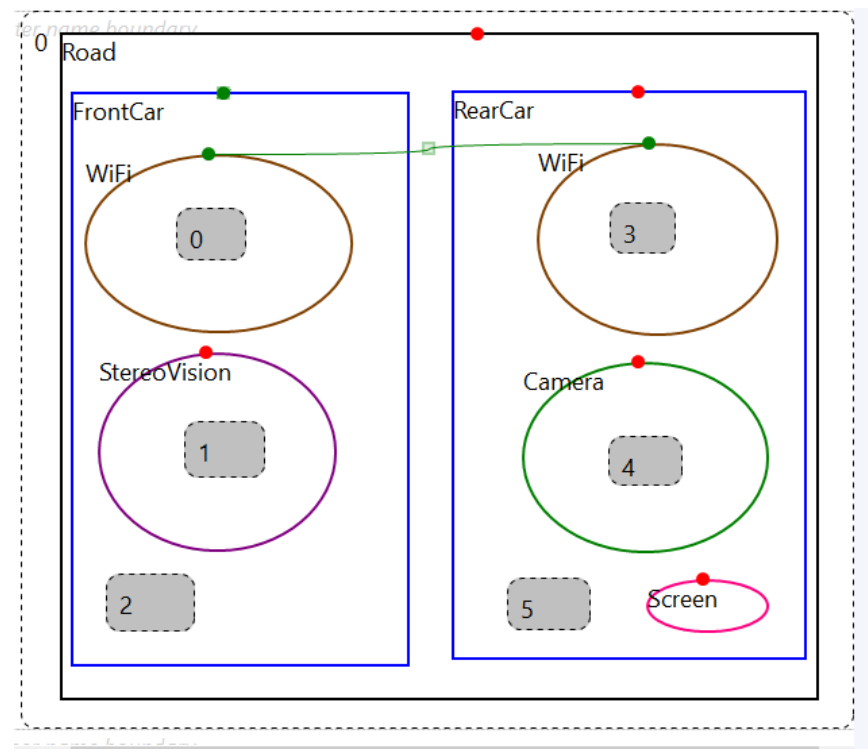

Figure 9. overtaking scenario-right side of the rule

or connect to others with converting this outer name to an edge. Here, when the rear car and front car are accessible by their wifi and Identify it as a valid user, they connect together in order to take advantages of Stereo vision placed in the front car and finally generate the augmented reality field of view for the rear car. In Figure 9, these two car converting outer name "b" to an edge that connect wifi of front car to the rear car.

The algebraic expression of the bigraphic model given in Figure 8 is as follows.

b/dFrontCar.(wifi.d $d_{0} \mid$ StereoVision. $d_{1} \mid d 2$ )

||RearCar.(wifi.d $d_{3} \mid$ Camera.d4 $\mid d_{5}$ )

The algebraic expression of the above context-aware reaction rule is as follows.

Overtaking Reaction Rule:

b/dFrontCar.(wifi $i_{b} . d_{0} \mid$ StereoVision. $\left.d_{1} \mid d 2\right)$

$\|$ RearCar.(wifi $i_{d} . d_{3} \mid$ Camera.d4| $\left.\mid d_{5}\right) \rightarrow$

FrontCar.(wifi. $\mathrm{d}_{0} \mid$ StereoVision. $d_{1} \mid d 2$ )

||RearCar.(wifi.d. ${ }_{3} \mid$ Camera.d4|

screen.augmented $\left.\mid d_{5}\right)$

Here, we will explain the rule: $\mathrm{WiFi}$ in the front car has an outer name "b" and Wifi in the rear car has an outer name "d". So, they can talk each other. The left side of rule express the current situation of the rear car and the front car before connecting via their wifi's ports. The left side of the rule will be happen when two wifi connect together and make an edge between themselves. When they connect, the screen in the rear car displays the augmented field of view sent by Stereovision of the front car via the wifi.

\section{Discussion and Future Direction}

We reach to this point that we can express large number of safety/security scenarios with the bigraph reactive system. While, when the model is large enough it is difficult to trace the rules and models based on the algebraic expression, the bigraphical reactive system can help us to visualize the rules and models based on the bigraph theory. This is the positive part of using the BRS to formalize the context-awareness system. However, There is not a good tools to support the bigraph theory. While We used the Big Red tools introduced in the background section, there is a feeling of intense need to an appropriate tools that fully support the bigraph theory. Moreover, in the augmented reality application we switch to the different environment very much and it cause to complicate the model. Also, some details cannot show with the element of bigraph system such as time and threshed. For Future work, we are going to explore how we can formally check the different specs of a bigraph model such as a smart car. Furthermore, investigating how the bigraph system can support time and threshed can be a possible direction for our future work.

\section{Conclusion}

The bigraph Reactive System introduced by Miller[9] is one of the useful theory to formalize the ubiquitous computing. In this study, we investigate how we can formalize the context-awareness of an augmented reality system, here, a smart car. we considered the overtaking scenario as the safety/security related scenario and formalize it with using the BRS theory. We conclude that the BRS can formalize many safety/security scenarios in an augmented reality application. However, some scenarios related to time and threshold and having details can cause difficulty to use it.

\section{References}

[1] Weiser, Mark. "The Computer for the 21 st Century." Scientific american 265, no. 3 (1991): 94-105.

[2] B. Schilit; N. Adams; R. Want. (1994). "Context-aware computing applications". IEEE Workshop on Mobile Computing Systems and Applications (WMCSA'94), Santa Cruz, CA, US. pp. 89101.

[3] Dey, Anind K. (2001). "Understanding and Using Context". Personal Ubiquitous Computing. 5 (1): 47.

[4] Abowd, Gregory D., Anind K. Dey, Peter J. Brown, Nigel Davies, Mark Smith, and Pete Steggles. "Towards a better understanding of context and context-awareness." In International Symposium on Handheld and Ubiquitous Computing, pp. 304-307. Springer, Berlin, Heidelberg, 1999.

[5] Kaltz, J.W.; Ziegler, J.; Lohmann, S. (2005). "Context-aware Web Engineering: Modeling and Applications" (PDF). Revue d'Intelligence Artificielle. 19 (3): 439458.

[6] Schuettel, Patrick. "The Concise Fintech Compendium." Fribourg: School of Management Fribourg/Switzerland (2017).

[7] P. Zimmer, A Calculus for Context-awareness. BRICS Report Series RS-05-27, Aarhus, Denmark, 21 pages, 2005.

[8] F. Siewe, A. Cau, and H. Zedan, The Calculus of Context-aware Ambients, Journal of Computer and System Sciences, vol. 77 (4), Jul. 2011, pp. 597-620, doi:10.1016/j.jcss.2010.02.003

[9] R. Milner, The Space and Motion of Communicating Agents, Cambridge: Cambridge University Press, 2009.

[10] Milner, Robin. "Bigraphical reactive systems." In International Conference on Concurrency Theory, pp. 16-35. Springer, Berlin, Heidelberg, 2001. 
[11] Faithfull, Alexander John, Gian Perrone, and Thomas T. Hildebrandt. "Big red: A development environment for bigraphs." Electronic Communications of the EASST 61 (2013).

[12] Francois Siewe, Antonio Cau and Hussein Zedan, The Calculus of Context-aware Ambients, Journal of Computer and System Sciences, Volume 77, Issue 4, July 2011, Pages 597-620, JCSS IEEE AINA 2009.

[13] G. Roman, C. Julien, and J. Payton, A Formal Treatment of ContextAwareness, in FASE, ser. LNCS, no. 2984. Springer, 2004, pp. 1236. [13]

[14] Isabel Cafezeiro, et al. A Formal Framework for Modeling ContextAware Behavior in Ubiquitous Computing. ISoLA 2008, CCIS 17, pp. 519533, 2008.

[15] L. Birkedal, S. Debois, E. Elsborg, T. Hildebrandt, and H. Niss, Bigraphical Models of Context-Aware Systems, L. Aceto, and A. Ingolfsdottir (Eds.): FOSSACS 2006, LNCS 3921, pp. 187201, 2006

[16] Wang, Ju-Shu, Dong Xu, and Zhou Lei. "Formalizing the structure and behavior of context-aware systems in bigraphs." In Software and Network Engineering (SSNE), 2011 First ACIS International Symposium on, pp. 89-94. IEEE, 2011.

[17] Cherfia, Taha Abdelmoutaleb, Kamel Barkaoui, and Faza Belala. "A brs-based modeling approach for context-aware systems: A case study of smart car system." In Embedded and Ubiquitous Computing (EUC), 2014 12th IEEE International Conference on, pp. 310-314. IEEE, 2014.

[18] https://seriousaccidents.com/legal-advice/top-causes-of-car-accidents/

[19] Rameau, Franois, Hyowon Ha, Kyungdon Joo, Jinsoo Choi, Kibaek Park, and In So Kweon. "A real-time augmented reality system to see-through cars." IEEE transactions on visualization and computer graphics 22, no. 11 (2016): 2395-2404. 